\title{
Is a low FODMAP diet dangerous?
}

\author{
M. Bellini ${ }^{1} \cdot$ A. Rossi ${ }^{2}$ \\ Received: 26 June 2018 / Accepted: 26 June 2018 / Published online: 6 August 2018 \\ c) Springer Nature Switzerland AG 2018
}

Patients with irritable bowel syndrome (IBS) often identify food as one of the most common precipitants of their symptoms, significantly influencing their quality of life [1]. More than $60 \%$ of patients with IBS have already independently limited or excluded some food items from their diet before referral to a gastroenterologist [2].

Many diets are recommended without any proven efficacy to (presumed) IBS patients by non-professionals through television, newspapers, magazines and the Internet. The stranger and more bizarre the diet, the more popular it is.

It seems that many patients are looking for the magic recipe for a happy gut and almost every gastroenterological visit ends with the question "Doctor what should I eat?" Therefore, it is not surprising that dietary suggestions, mainly elimination diets, together with lifestyle recommendations, are the most frequent therapies prescribed by gastroenterologists to their IBS patients [3].

Are dietary suggestions we make really effective? Probably not on the basis of evidence-based medicine and according to the British Dietetic Association: the recommendation level for most elimination diets is between $C$ and D. Only the low FODMAP diet (LFD) reaches evidence level B [4].

The acronym FODMAP stands for fermentable oligo- diand mono-saccharides and polyols. FODMAPs are small molecules, containing only 1-10 sugars. They can be found in a wide range of very common foods such as fruit, vegetables, legumes and cereals, honey, milk and dairy products, sweeteners and so on. Total daily intake of FODMAPs, in a usual diet, ranges from 15 to $30 \mathrm{~g}$ per day (Table1).

FODMAPs' mechanisms of action are linked to their osmotic activity, forcing water into the gastrointestinal tract. Moreover, after entering the colon, they are a good source

M. Bellini

mbellini58@gmail.com

1 Gastrointestinal Unit, Department of Surgery, University of Pisa, Pisa, Italy

2 Division of Rheumatology, Department of Clinical and Experimental Medicine, University of Pisa, Pisa, Italy of food for the intestinal microbiota, which ferment them, increasing hydrogen, methane and carbon dioxide production, which in turn increase luminal distension. This, in the context of the visceral hypersensitivity typical of IBS patients, may provoke abdominal pain, bloating, flatulence and alterations in bowel habits [5, 6].

Other mechanisms by which FODMAPs can be harmful are an excessive production of short chain fatty acids (SCFAs) able to increase visceral hypersensitivity and stimulate the production of histamine (provoking a neuroinflammatory response involving mast cell activation) and the release of 5 HT from intestinal mucosa, leading to modified intestinal motility, secretion and sensitivity and, therefore, exacerbating IBS symptoms [7].

At least 10 randomized controlled trials testing the LFD in IBS patients have been published. In eight of them it is demonstrated to be an effective treatment in the short term in up to $76 \%$ of patients, at least for some of the primary and/or secondary endpoints investigated. In two of them LFD was no better than comparators (hypnotherapy and NICE dietetic guidelines for IBS, respectively) [8].

Are FODMAPs dangerous? Not at all, because they increase stool bulk and favor calcium absorption and decrease serum lipid levels. They have a prebiotic effect, favoring the growth of beneficial bacteria (namely Bifidobacteria) and increasing the production of SCFAs such as butyrate, the most important energy source for the colonocyte and also propionate and acetate, which may have systemic immunomodulatory and epigenetic effects [9].

Because of these beneficial effects of FODMAPs, the LFD has been sometimes regarded as harmful and some potential limitations and concerns regarding this diet remain to be clarified. LFD detractors maintain that it is complex and difficult to teach and learn, expensive and difficult to continue and can induce dangerous changes in intestinal microbiota and SCFA production [8]. Moreover, they claim it could be nutritionally inadequate (inducing excessive weight loss, reduction of serum iron, calcium and vitamins) and could lead particularly predisposed patients to an eating behavior disorder [8]. 


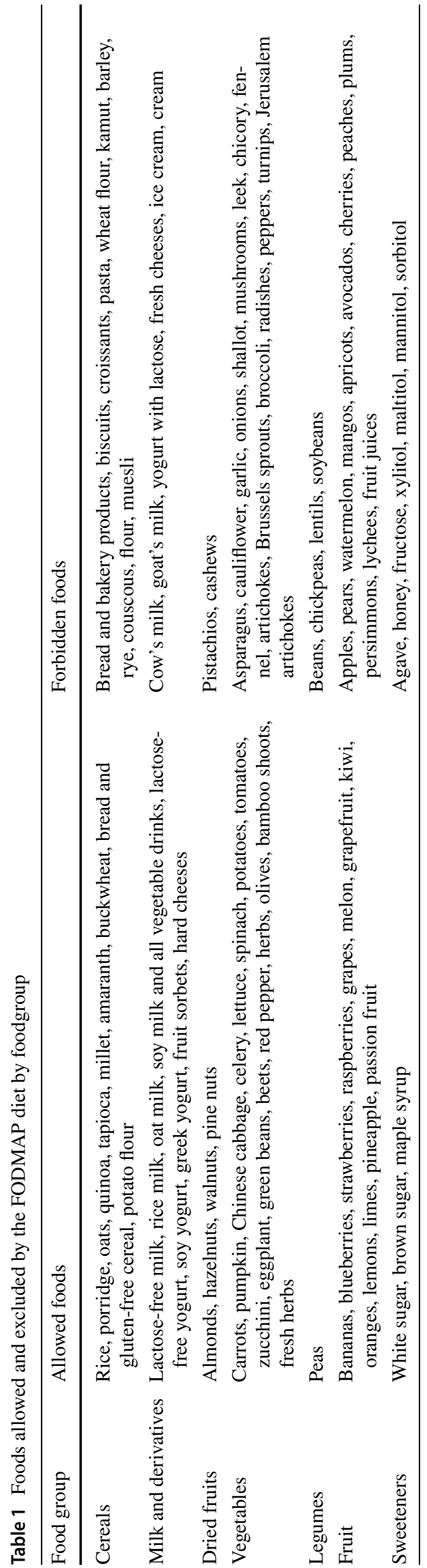

These concerns may be justified in patients who follow the diet without any professional advice [10] because an LFD has to be prescribed by health care professionals (nutritionists, dieticians and/or gastroenterologists) who have skills and expertise in nutritional issues and sufficient time to teach a diet, without resorting to non-personalized diet sheets which are often out of date [10].

Another frequent criticism is in regard to the difficulty in continuing a LFD and its costs. However, O' Keeffe et al. [11], in a recent study on 103 patients, with a follow-up of 6-18 months, found that LFD acceptability and food-related quality of life seemed to be good. Patients only found it a little more expensive than their usual diet and met some difficulties eating out at restaurants, at friends' houses and when traveling.

Probably the most complex issue regarding LFD, which is still subject to debate, is the possible change of the intestinal microbiota and metabolome. There are at least seven studies which have examined this problem $[8,12]$. The outcomes of these studies are very different and often conflicting. A reduction in relative and absolute abundance of Bifidobacteria and a lower production of SCFAs (as confirmed by a lower stool concentration) are possible. Unfortunately, all the studies examined the microbiome and the metaboloma of IBS patients at the end of the strict LFD period (recommended for no more than 8 weeks) and not after the reintroduction phase, that is the moment when non-harmful foods are reintroduced and a more relaxed, less restrictive diet, the "adapted LFD", starts.

This issue is strictly linked to the criticism of LFD nutritional adequacy. In a paper we have recently published in this journal, we evaluated mean dietary intake [13]. The amount of calories, proteins, fats, carbohydrates and fibers slightly decreased during the 8 weeks of LFD but without significant changes in comparison with the values before starting the LFD. Anthropometric data showed no significant change and in no patient did the LFD induce a change in nutritional blood test, results. These results were confirmed by bioelectric vector impedance analysis where again, no significant changes were observed after the 8 weeks of LFD [13].

The most important matter of debate is the efficacy of a LFD in the medium to long term. Data regarding this issue are still scarce and there are currently only two studies in the literature that deal with this topic. Shepherd et al. [14], at 14-month follow-up, found $77 \%$ of patients adherent to the diet with $76 \%$ reporting significant improvement in abdominal symptoms. More recently, O'Keeffe et al. [11], in 103 IBS patients, with a follow-up of 6-18 months, found significant reductions in abdominal pain, bloating, flatulence, incomplete evacuation, and lethargy, with pain, bloating and flatulence decreasing by more than one-third in the long term. 
A strict LFD is not recommended for a long period and a more balanced, less restrictive diet (adapted LFD) should be started within no more than 8 weeks. The impact on the intestinal microbiota and metabolome has to be further investigated. Larger randomized controlled trials testing the efficacy and safety of the adapted LFD and patients' adherence to it are needed.

\section{Compliance with ethical standards}

Conflict of interest The authors declare that they have no conflict of interest.

Ethical approval This article does not contain any studies with human participants performed by any of the authors.

Informed consent For this type of study formal consent is not required.

\section{References}

1. Thomas A, Quigley EM (2015) Diet and irritable bowel syndrome. Curr Opin Gastroenterol 31(2):166-171. https://doi.org/10.1097/ MOG.0000000000000158

2. Monsbakken KW, Vandvik PO, Farup PG (2006) Perceived food intolerance in subjects with irritable bowel syndrome-etiology, prevalence and consequences. Eur J Clin Nutr 60(5):667-672

3. Bellini M, Usai-Satta P, Bove A, Bocchini R, Galeazzi F, Battaglia E, Alduini P, Buscarini E, Bassotti G, ChroCoDiTE Study Group, AIGO (2017) Chronic constipation diagnosis and treatment evaluation: the "CHRO.CO.DI.T.E." study. BMC Gastroenterol 17(1):11. https://doi.org/10.1186/s12876-016-0556-7

4. McKenzie YA, Bowyer RK, Leach H, Gulia P, Horobin J, O'Sullivan NA, Pettitt C, Reeves LB, Seamark L, Williams M, Thompson J, Lomer MC, IBS Dietetic Guideline Review Group on behalf of Gastroenterology Specialist Group of the British Dietetic Association (2016) British Dietetic Association systematic review and evidence-based practice guidelines for the dietary management of irritable bowel syndrome in adults (2016 update). J Hum Nutr Diet 29(5):549-575. https://doi.org/10.1111/ jhn. 12385

5. Barrett JS, Gearry RB, Muir JG, Irving PM, Rose R, Rosella O, Haines ML, Shepherd SJ, Gibson PR (2010) Dietary poorly absorbed, short-chain carbohydrates increase delivery of water and fermentable substrates to the proximal colon. Aliment Pharmacol Ther 31(8):874-882. https://doi.org/10.111 1/j.1365-2036.2010.04237.x

6. Ong DK, Mitchell SB, Barrett JS, Shepherd SJ, Irving PM, Biesiekierski JR, Smith S, Gibson PR, Muir JG (2010) Manipulation of dietary short chain carbohydrates alters the pattern of gas production and genesis of symptoms in irritable bowel syndrome. J Gastroenterol Hepatol 25(8):1366-1373. https://doi.org/10.111 1/j.1440-1746.2010.06370.x

7. Hill P, Muir JG, Gibson PR (2017) Controversies and recent developments of the low-FODMAP diet. Gastroenterol Hepatol (NY) 13(1):36-45

8. Staudacher HM, Whelan K (2017) The low FODMAP diet: recent advances in understanding its mechanisms and efficacy in IBS. Gut 66(8):1517-1527. https://doi.org/10.1136/gutjnl-2017-31375 0

9. Catassi G, Lionetti E, Gatti S, Catassi C (2017) The low FODMAP diet: many question marks for a catchy acronym. Nutrients 9(3):292. https://doi.org/10.3390/nu903029

10. Gibson PR, Burgell RE (2017) Easing concerns about the low FODMAP diet in patients with irritable bowel syndrome. Gastroenterology 153(4):886-887. https://doi.org/10.1053/j.gastr o.2017.08.055

11. O'Keeffe M, Jansen C, Martin L, Williams M, Seamark L, Staudacher HM, Irving PM, Whelan K, Lomer MC (2018) Long-term impact of the low-FODMAP diet on gastrointestinal symptoms, dietary intake, patient acceptability, and healthcare utilization in irritable bowel syndrome. Neurogastroenterol Motil. https://doi. org/10.1111/nmo.13154

12. Harvie RM, Chisholm AW, Bisanz JE, Burton JP, Herbison P, Schultz K, Schultz M (2017) Long-term irritable bowel syndrome symptom control with reintroduction of selected FODMAPs. World J Gastroenterol 23(25):4632-4643. https://doi.org/10.3748/ wjg.v23.i25.4632

13. Bellini M, Gambaccini D, Bazzichi L, Bassotti G, Mumolo MG, Fani B, Costa F, Ricchiuti A, de Bortoli N, Mosca M, Marchi S, Rossi A (2017) Bioelectrical impedance vector analysis in patients with irritable bowel syndrome on a low FODMAP diet: a pilot study. Tech Coloproctol 21(6):451-459. https://doi.org/10.1007/ s10151-017-1639-3

14. Shepherd SJ, Gibson PR (2006) Fructose malabsorption and symptoms of irritable bowel syndrome: guidelines for effective dietary management. J Am Diet Assoc 106:1631-1639 